\title{
Congenital Erythropoietic Porphyria: Mutation of the Uroporphyrinogen III Cosynthase Gene in a Vietnamese Patient
}

\author{
Dao Hoang Thien Kim ${ }^{a} \quad$ Asako Kawazoe $^{a} \quad$ Pham Dang Bang ${ }^{b}$ \\ Nguyen Tien Thanh ${ }^{c}$ Shigeru Taketani ${ }^{a}$ \\ ${ }^{a}$ Department of Biotechnology, Kyoto Institute of Technology, Kyoto, Japan; ${ }^{b}$ Department \\ of Dermatology, Hanoi Medical University, and ${ }^{C}$ National Institute of Dermatology and \\ Venereology, Hanoi, Vietnam
}

\section{Key Words}

Congenital erythropoietic porphyria · Uroporphyrinogen III cosynthase · Deficiency · Photosensitivity · Mutation

\begin{abstract}
Congenital erythropoietic porphyria (CEP) arises from an autosomal recessive inherited disorder of the porphyrin metabolism, which leads to the accumulation of uroporphyrinogen I in bone marrow, skin and several other tissues by a deficiency of uroporphyrinogen III cosynthase (UROS). We studied a Vietnamese patient and her family suffering from severe cutaneous photosensitivity with skin fragility, bullous lesions and hypertrichosis on lightexposed areas. A missense mutation in the UROS gene was identified as a transversion of $G$ to $T$ at nucleotide 11,776, resulting in a substitution of valine by phenylalanine at codon 3 of exon 2. The patient showed a homozygous mutant profile, and the heterozygous state was observed in the parents. The activity of mutated UROS expressed in Escherichia coli was less than $16.1 \%$ that of the control, indicating that the markedly reduced activity of UROS is responsible for CEP. We described for the first time a mutation in the UROS gene in a Southeast Asian patient and a molecular diagnosis for the identification of clinically asymptomatic heterozygous mutation carriers and families with CEP.
\end{abstract}




\section{Introduction}

Congenital erythropoietic porphyria (CEP) is transmitted as an autosomal recessive trait in which the activity of uroporphyrinogen III cosynthase (UROS) is severely reduced. The disease is biochemically characterized by the accumulation of excess uroporphyrinogen I and coproporphyrin in plasma, tissues and red cells [1,2], and by the excretion of these porphyrins in urine and feces. CEP is clinically characterized by severe photosensitivity with skin fragility, bullous lesions, hypertrichosis and scarring on light-exposed areas. Individuals with CEP can be either homozygous with the same mutation or compound heterozygous, and the severity of the disease is inversely related to the residual enzyme activity of UROS [1-3]. Here, we report a mutation of the UROS gene in a patient with CEP who is the first Vietnamese case. The mutation in the UROS gene found in the patient caused reduced enzyme activity and is the same as that previously identified in a Japanese patient.

\section{Case Report}

We report on a 5-year-old girl with a history of pink-stained urine as a newborn. Disease onset occurred in the tenth month of life. Her deciduous teeth sprouted with a red-brown color. She also suffered from episodes of burning sensation, erythema and edema on her face, neck, arms and lower legs within minutes following exposure to direct sunlight in her infancy. Vesicles or subepidermal bullous lesions progressed to crusted erosions, which healed with scarring and either hyperpigmentation or hypopigmentation (fig. 1a). They appeared constantly and got worse during the spring and summer months. Hypertrichosis developed on unprotected skin areas. Spleen enlargement was also observed. Urine porphyrins were extremely elevated to $16 \mu \mathrm{mol} / \mathrm{dl}$ with a predominance of coproporphyrins and uroporphyrins. However, the detailed concentration of each porphyrin was not analyzed. Severe cutaneous photosensitivity, blistering of light-exposed skin, history of pinkstained diapers and accumulation of porphyrins in the blood and various other tissues, particularly erythrodontia (fig. 1b), suggested the diagnosis of CEP. The clinical and biochemical data of the patient are summarized in table 1 . With strict avoidance of UV and visible light, use of topical sunscreen, oral treatment with $\beta$-carotene and antibiotic for preventing bacterial infection of the skin, the bullae healed, leaving milia and hyperpigmented scars without the need of a blood transfusion.

Mutation analysis of the UROS gene of the patient with CEP was performed. The DNA of exons 2-10 including splicing junctions of all exons was amplified by PCR and then sequenced. A single missense mutation corresponding to a G-to-T transversion $(11,776 \mathrm{~g}>\mathrm{t})$ in exon 2 was found, leading to an amino acid change from valine at position 3 to phenylalanine (fig. 2). The result indicated that the patient was homozygous for this mutation. Then, we also analyzed the UROS genes of her parents who were shown to be heterozygote carriers of this mutation (fig. 3). Her brother had died of a disease similar to hers in terms of symptoms and abnormalities, suggesting that he had the same gene mutation (V3F). Conversely, on the basis of the pedigree analysis spanning three generations, no other symptomatic patients were found. To confirm the genotype-phenotype relationship, we constructed a pET-duet vector [4] carrying mutated cDNA of UROS and transformed it into an Escherichia coli BL21 strain. The expression of UROS in bacteria was induced at $25^{\circ} \mathrm{C}$. After purification of the expressed enzyme with nickel ion-beads (Qiagen Inc., Valencia, Calif., USA), the activity of mutated UROS (V3F) was compared with that of normal enzyme by the 
method of Wright and Lim [5]. The specific activity of the mutated UROS was less than $16.1 \%$ that of the control.

\section{Discussion}

In this study, the mutational analysis of the UROS gene revealed a mutation (V3F) in the genomic DNA of a CEP patient from Vietnam. The patient was homozygous for the missense mutation in $\mathrm{V} 3 \mathrm{~F}$ and her parents were shown to be heterozygous carriers of the same mutation. No further family members were available for molecular analysis, but her brother had already died in association with symptoms similar to those of the patient. The family was hesitant to undergo additional biochemical studies to assess residual enzymatic activity to expand the genotype-phenotype assessment. The deficiency, but not absence, of UROS results primarily in the accumulation of porphyrins in various tissues. The complete absence of UROS would likely lead to death. We constructed mutated cDNA and expressed the recombinant protein in E. coli. The mutated UROS (V3F) showed low enzyme activity with $16.1 \%$ of the control. Although the age of onset and clinical manifestations in CEP are highly variable, from severe to milder forms of hemolytic anemia with only cutaneous lesions among adult CEP patients, photosensitivity was observed soon after birth in this patient. Furthermore, severe clinical manifestations in infancy were observed in this patient, which were derived from the reduced enzymatic activity of UROS. Substitution of valine with phenylalanine at position 3 causes a change of the side chain of the hydrophobic amino acid, which likely leads to structural alterations at the N-terminus of UROS [6]. This mutation has been previously described in a Japanese patient [3] and a L4F mutation was found in American patients [1], suggesting that the $\mathrm{N}$-terminal region of the enzyme is quite important for the catalytic reaction. The amino acid sequence MKVLLL at the $\mathrm{N}$-terminus of UROS is well conserved among mammals and many other organisms, which also supports the assertion that it is an indispensable domain. Based on the 3D structure of UROS [6], the conserved amino acids at the $\mathrm{N}$-terminus form a $\beta$-sheet associated with domains of $\alpha$-helix and other $\beta$-sheets of the enzyme and may play an important role in the maintenance of the basic structure of UROS. The present study showed that the change of the isopropyl group from valine to the phenyl group of phenylalanine markedly reduced the enzyme activity, and other investigators reported that the change of the isopropyl group from leucine to a phenyl group at the next amino acid (L4F) also reduced the activity [1]. Thus, small structural alterations such as changes of a hydrophobic-hydrophobic group in the N-terminus of the protein can result in a dramatic reduction in enzyme activity.

CEP is a genetic disease for which therapy includes avoidance of visible light, especially the Soret band wavelengths $(400-410 \mathrm{~nm})$, and blood transfusions to decrease porphyrin production. The treatment modalities, such as zinc sunscreens, oral $\beta$-carotene, oral charcoal and other porphyrin binders, have been prescribed with limited effects [1]. On the contrary, allogeneic bone marrow transplantation (BMT) has been successful for the treatment of CEP [7]. It has been reported that BMT in several patients resulted in a decrease in porphyrin levels and photosensitivity reduction [7]. Moreover, BMT significantly reduces splenomegaly and porphyrin excretion, and it decreases the cutaneous lesions caused by sun exposure in CEP patients [7]. Therefore, BMT is one of the most rational therapies for CEP patients, although it needs adjustment for HLA-matched donors. Conversely, it should be noted that patients with CEP face increased risk of graft rejection when a donor is HLA-mismatched, and then the risk of BMT must be balanced with the severity of the disease. In the future, the 
use of stem cells engineered with vector-mediated expression of a correct copy of the UROS cDNA can be a treatment option [8].

At the time of writing, about 40 different mutations have been reported in UROS. Among them, C73R is the most frequent mutation leading to a severe phenotype in CEP patients. This mutation has been observed in one third of CEP patients and in half of the disease alleles in European countries [1]; however, it has not been reported in Asian countries. L237P and S47P have been observed in Western Asia [9]. Six UROS mutations were found in Japanese CEP patients, in whom V3F was observed in the homozygote state. All other studied patients were either compound heterozygotes or homozygous for other mutations in the middle of the primary structure of UROS [6]. This is the first analysis of a CEP patient in Southeast Asia, and no further data of molecular defects in the UROS gene are currently available in relation to the genotype of the UROS gene in this area. The V3F mutation has not been identified in Caucasian populations, but its presence was found in a Japanese patient. The reason for the presence of the same V3F mutation in Vietnamese and Japanese patients is not clear since these countries are far apart and separated by the sea; however, a common ancestry between these countries may be suggested.

\section{Acknowledgement}

We thank Drs. H. Sato and M. Noguchi for the kind gift of bacterial expression plasmid carrying HMBS cDNA.

This study was supported in part by grants from the Ministry of Health, Labor and Welfare of Japan, and from the Ministry of Education, Science, Sports and Culture of Japan.

\section{Disclosure Statement}

None declared.

\section{References}

1 Desnick RJ, Astrin KH: Congenital erythropoietic porphyria: advances in pathogenesis and treatment. Br J Haematol 2002;117:779-795.

-2 Wiederholt T, Poblete-Gutiérrez P, Gardlo K, Goerz G, Bolsen K, Merk HF, Frank J: Identification of mutations in the uroporphyrinogen III cosynthase gene in German patients with congenital erythropoietic porphyria. Physiol Res 2006;55(suppl 2):S85-S92.

-3 Takamura N, Hombrados I, Tanigawa K, Namba H, Nagayama Y, de Verneuil H, Yamashita S: Novel point mutation in the uroporphyrinogen III synthase gene causes congenital erythropoietic porphyria of a Japanese family. Am J Med Genet 1997;70:299-302.

-4 Ohgari Y, Sawamoto M, Yamamoto M, Kohno H, Taketani S: Ferrochelatase consisting of wild-type and mutated subunits from patients with a dominant-inherited disease, erythropoietic protoporphyria, is an active but unstable dimer. Hum Mol Genet 2005;14:327-334.

5 Wright DJ, Lim CK: Simultaneous determination of hydroxymethylbilane synthase and uroporphyrinogen III synthase in erythrocytes by high-performance liquid chromatography. Biochem J 1983;213:85-88.

-6 Mathews MA, Schubert HL, Whitby FG, Alexander KJ, Schadick K, Bergonia HA, Phillips JD, Hill CP: Crystal structure of human uroporphyrinogen III synthase. EMBO J 2001;20:5832-5839.

7 Katugampola RP, Anstey AV, Finlay AY, Whatley S, Woolf J, Mason N, Deybach JC, Puy H, Ged C, de Verneuil H, Hanneken S, Minder E, Schneider-Yin X, Badminton MN: A management algorithm for congenital erythropoietic porphyria derived from a study of 29 cases. Br J Dermatol 2012;167:888-900.

-8 Mazurier F, Géronimi F, Lamrissi-Garcia I, Morel C, Richard E, Ged C, Fontanellas A, Moreau-Gaudry F, Morey M, de Verneuil H: Correction of deficient CD34+ cells from peripheral blood after mobilization in a patient with congenital erythropoietic porphyria. Mol Ther 2001;3:411-417. 
Kim et al.: Congenital Erythropoietic Porphyria: Mutation of the Uroporphyrinogen III Cosynthase Gene in a Vietnamese Patient

-9 Moghbeli M, Maleknejad M, Arabi A, Abbaszadegan MR: Mutational analysis of uroporphyrinogen III cosynthase gene in Iranian families with congenital erythropoietic porphyria. Mol Biol Rep 2012;39:67316735 .

Table 1. Clinical and biochemical features in a patient with CEP

\begin{tabular}{lllc}
\hline Clinical features & \multicolumn{3}{l}{ Biochemical features } \\
\hline Anemia & + & RBC & $4.78 \mathrm{million} / \mathrm{mm}^{3}$ \\
Splenomegaly & + & HCT & $34.6 \%$ \\
Erythrodontia & + & Hemoglobin & $10.3 \mathrm{~g} / \mathrm{dl}$ \\
Hypertrichosis & face & MCH & $22.2 \mathrm{pg}$ \\
& & Urine total porphyrins & $16 \mu \mathrm{mol} / \mathrm{dl}$ \\
& & control & $<10 \mathrm{nmol} / \mathrm{dl}$ \\
Skin photosensitivity & severe cutaneous lesions, blistering, & & \\
& scarring & & \\
Erythrodontia & + & & \\
Hyperpigmentation & + & & \\
Hemolysis & \pm & & \\
Liver function & normal & & \\
\hline
\end{tabular}
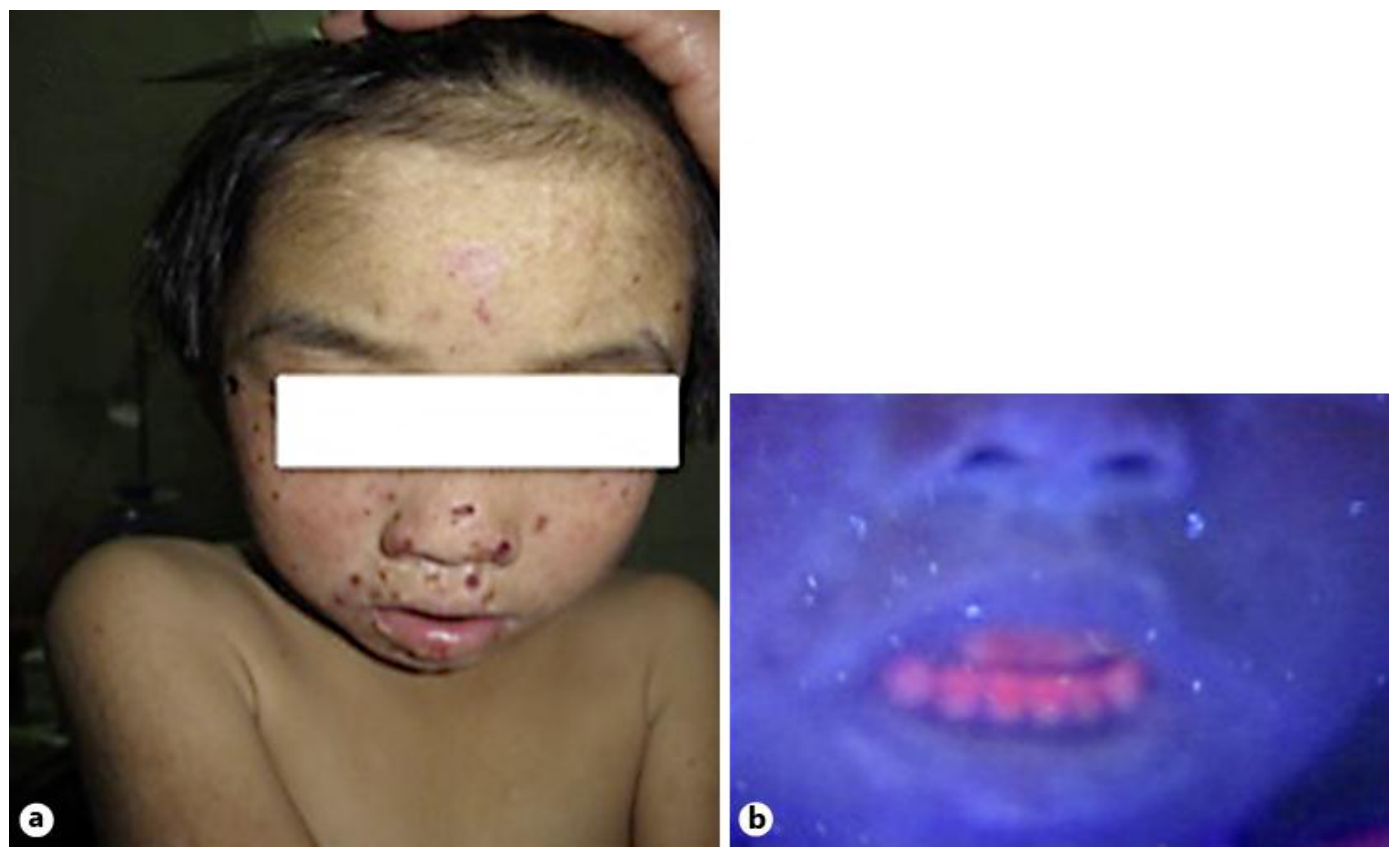

Fig. 1. Photograph of a patient with CEP. a Crusted erosions and hypertrichosis on the forehead. b Redbrown teeth with intense red fluorescence under WOOD's light detection. 


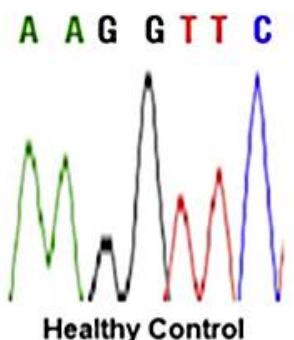

Healthy Control

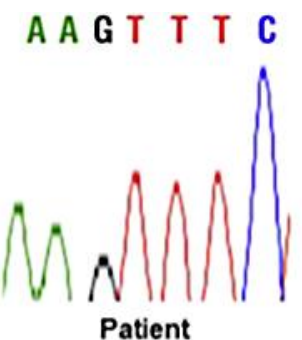

A A G G/T T T C

A A G G/T T T C

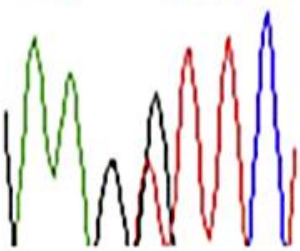

Father

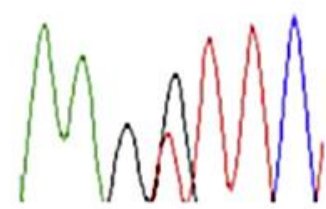

Mother

Fig. 2. Nucleotide sequences of exon 2 of the UROS gene of the patient, her parents and a healthy control. Missense V3F mutation comprising a homozygous G-to-T transversion.

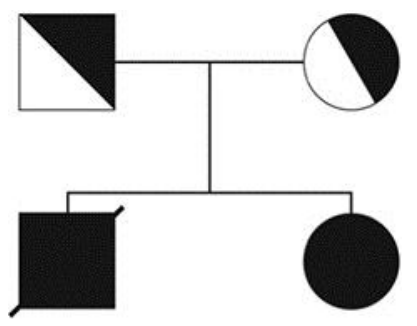

Fig. 3. Family pedigree of the CEP patient. The patient was homozygous for V3F. Her brother had died of a disease similar to that of the patient, and her parents were asymptomatic heterozygotes. 